\title{
BILATERAL WEIGHTED SHIFT OPERATORS SIMILAR TO NORMAL OPERATORS
}

\author{
GYÖRGY PÁL GEHÉR
}

Abstract. We prove that an injective, not necessarily bounded weighted bilateral shift operator on $\ell^{2}(\mathbb{Z})$ is similar to a normal operator if and only if it is similar to a scalar multiple of the simple (i.e. unweighted) bilateral shift operator $S$.

Mathematics subject classification (2010): 47B37, 47B15.

Keywords and phrases: Bilateral weighted shift operator, similarity to normal operators.

\section{REFERENCES}

[1] N. E. Benamara And N. Nikolski, Resolvent tests for similarity to a normal operator, Proc. London Math. Soc. 78, no. 3 (1999), 585-626.

[2] J. B. Conway, A Course in Functional Analysis, Springer-Verlag, New York, 1990.

[3] S. R. Foguel, A counterexample to a problem of Sz.-Nagy, Proc. Amer. Math. Soc. 15, no. 5 (1964), 788-790.

[4] Gy. P. GeHÉR, Asymptotic limits of operators similar to normal operators, Proc. Amer. Math. Soc. 143, 11 (2015), 4823-4834.

[5] P. R. Halmos, Ten problems in Hilbert space, Bull. Amer. Math. Soc. 76, no. 5 (1970), 887-933.

[6] C. S. Kubrusly, An Introduction to Models and Decompositions in Operator Theory, Birkhäuser, Boston, 1997.

[7] S. KUPIN, Linear resolvent growth test for similarity of a weak contraction to a normal operator, Ark. Mat. 39, no. 1 (2001), 95-119.

[8] S. KUPIN, Operators similar to contractions and their similarity to a normal operator, Indiana Univ. Math. J. 52, no. 3 (2003), 753-768.

[9] A. LeBow, A power-bounded operator that is not polynomially bounded, Michigan Math. J. 15, no. 4 (1968), 397-399.

[10] N. K. NIKOLSKI, Treatise on the Shift Operator, Springer-Verlag, Berlin, 1986.

[11] G. PISIER, A polynomially bounded operator on Hilbert space which is not similar to a contraction, J. Amer. Math. Soc. 10, no. 2 (1997), 351-369.

[12] S. ÔTA AND K. SCHMÜDGEN, On some classes of unbounded operators, Integr. Equat. Oper. Th. 12, no. 2 (1989), 211-226.

[13] H. N. Salas, Hypercyclic weighted shifts, Trans. Amer. Math. Soc. 347, no. 3 (1995), 993-1004.

[14] H. N. SAlas, Supercyclicity and weighted shifts, Studia Math. 135, no. 1 (1999), 55-74.

[15] A. L. SHIELDS, Weighted shift operators and analytic function theory, Topics in Operator Theory, Math. Surveys 13, Amer. Math. Soc., Providence, R. I. (1974), 49-128.

[16] B. Sz.-Nagy, C. Foias, H. Bercovici AND L. KéRChy, Harmonic Analysis of Operators on Hilbert Space, Springer, New York, 2010.

[17] B. SZ.-NAGY, On uniformly bounded linear transformations in Hilbert space, Acta Sci. Math. (Szeged) 11, no. 3-3 (1947), 152-157. 